\title{
Evaluación experimental de la solución analítica exacta de la ecuación de Colebrook-White
}

\section{Experimental evaluation of exact analytical solution of the Colebrook-White Equation}

\author{
Alfaro-Guerra Marco \\ Universidad de La Serena, Chile \\ Instituto de Investigación Multidisciplinario en Ciencia y Tecnología \\ Correo: malfarog@userena.cl \\ http://orcid.org/0000-0002-7238-2437
}

Guerra-Rojas Rodrigo

Universidad de La Serena, Chile

Facultad de Ingeniería

Correo:rguerra@userena.cl

http://orcid.org/0000-0002-4978-0074

\author{
Olivares-Gallardo Alan \\ Universidad de La Serena, Chile \\ Facultad de Ingeniería \\ Correo: aolivares@userena.cl \\ http://orcid.org/0000-0003-1473-2264
}

\section{Resumen}

En el presente trabajo se diseñó y construyó un sistema hidráulico para la determinación experimental del factor de fricción en una tubería de PVC bajo flujo turbulento. Este sistema permite conducir el agua por una tubería donde se midieron el caudal y la diferencia de presión para calcular la pérdida de carga entre dos puntos de control, considerando las propiedades asociadas a la tubería como es la rugosidad relativa, su diámetro y longitud. Utilizando la ecuación de Darcy-Weisbach se determinó el factor de fricción experimental. El objetivo del presente trabajo fue evaluar en forma experimental el factor de fricción y compararlo con el obtenido utilizando la fórmula n-ésima como aproximación de la solución exacta de la ecuación de Colebrook-White propuesta por Mikata y Walczak en 2015. La fórmula n-ésima predice el factor de fricción con un error relativo que tiende a disminuir dependiendo de la profundidad de la recursión. En nuestro análisis, para un valor de n igual a 15 el error en la determinación del factor de fricción reportó valores inferiores a 1E-16. Se desarrolló una macro de Excel en lenguaje VBA para evaluar la recursión dada la dificultad numérica de resolver el valor analítico exacto de la ecuación de Colebrook-White. La originalidad del presente trabajo corresponde a la evaluación de la fórmula n-ésima del factor de fricción y su comparación con los resultados experimentales obtenidos en el laboratorio, lo que permite probar la validez de la predicción de la fórmula con un caso real. Esto permitió encontrar el error relativo porcentual respecto al valor experimental.

Descriptores: Factor de fricción, solución analítica de la ecuación de Colebrook-White, caída de presión en tuberías, recursión infinita, sistema experimental.

\begin{abstract}
In the present work a hydraulic system was designed and built for the experimental determination of the friction factor in a PVC pipe under turbulent flow. This system allows the water to be conducted through a pipeline where the flow and the pressure difference were measured to calculate the pressure loss between two control points, considering the properties associated with the pipe such as the relative roughness, diameter and length. Using the Darcy-Weisbach equation, the experimental friction factor was determined. The objective of the present work was to experimentally evaluate the friction factor and compare it with that obtained using the nth formula as an approximation of the exact solution of the Colebrook-White equation proposed by Mikata and Walczak in 2015. The nth formula, this predicts the friction factor with a relative error that tends to decrease depending on the depth of the recursion. In our analysis, for a value of $\mathrm{n}$ equal to 15 , the error in the determination of the friction factor reported values lower than $1 \mathrm{E}-16$. An Excel macro was developed in VBA language to evaluate the recursion given the numerical difficulty of solving the exact analytical value of the Colebrook-White equation. The originality of this work corresponds to the evaluation of the nth formula of the friction factor and its comparison with the experimental results obtained in the laboratory, which allows to prove the validity of the prediction of the formula with a real case. This allowed finding the percentage relative error with respect to the experimental value.

Keywords: Friction factor, analytical solution of the Colebrook-White Equation, pressure drop in pipes, infinite recursion, experimental system.
\end{abstract}




\section{INTRODUCCIÓN}

En la actualidad, el manejo de los recursos hídricos es importante, por ello, los sistemas hidráulicos que distribuyen a los usuarios tienen un rol fundamental (Ángeles et al., 2000). En los sistemas hidráulicos, específicamente en las tuberías y diferentes singularidades, siempre se desarrolla fricción, ocasionando caídas de presión en el flujo a lo largo de su trayectoria. Es importante conocer esta caída de presión para una apropiada estimación de las presiones de trabajo en los sistemas hidráulicos. Por ello, se han efectuado diferentes estudios para determinar de manera precisa el factor de fricción en tuberías. (Anaya et al., 2014). En el diseño, la longitud de tuberías permanece como parámetro constante e independiente del factor de fricción en los distintos tramos de las redes de agua. En contraste, varios parámetros tales como el diámetro de la tubería y el factor de fricción varían durante la vida útil de las conducciones (Özger and Yildirim, 2009). Al realizar el análisis de las pérdidas de carga en los sistemas de distribución de agua, se debe tener en cuenta que el factor de fricción es calculado para tres estados del flujo, esto es, flujo laminar, transición y turbulento.

Muchos son los autores que han contribuido de manera fundamental al conocimiento de aspectos relevantes de la Ingeniería Hidráulica tanto en la era moderna como en los inicios del estudio de dichos fenómenos. Existe una propuesta gráfica como el Gráfico de Moo$\mathrm{dy}$, que es la figura más útil y conocida para determinar el factor de fricción de la pérdida de carga, la cual es fiable si se aceptan errores inferiores a $15 \%$ en cálculos de diseño (White, 2008). Al mismo tiempo, existe un número importante de propuestas de forma analítica, que describen algún fenómeno hidráulico y que perduran hasta el día de hoy. Otras propuestas han sufrido mejoras o modificaciones avaladas por la rapidez y precisión que se puede lograr con las soluciones numéricas y tecnológicas propias de la actualidad.

Por ello, se valora aún más el esfuerzo y aporte realizado por autores como Reynolds O., Nikuradse J., Prandtl L., Von Kármán T., Colebrook C.F., White C.M., Moody L.F., entre otros, que son citados recurrentemente con artículos que se publican en la materia, en revistas internacionales de investigación científica.

Como es de amplio conocimiento, Colebrook y White publicaron en el año 1937, en el Journal "The Royal Society", el artículo: "Experiments with Fluid Friction in Roughened Pipes" citando los trabajos del año 1933 de Nikuradse y Prandtl (Colebrook and White, 1937). En 1939 Colebrook publicó el trabajo denominado: "Turbulent Flow in Pipes, with particular reference to the Transition Region between the Smooth and Rough Pipe Laws." en the Institution Journal, el cual integra como parte principal: "A New Theorical Formula for Flow in the Transition Region". Esta fórmula teórica para flujos en la Región de Transición ha perdurado con el tiempo y se ha estudiado y citado por innumerables autores, es aceptada y validada como el valor más preciso en el cálculo del factor de fricción de tuberías hidráulicamente lisas y rugosas (Colebrook, 1939).

Moody en 1944 publicó el texto: "Friction Factors for Pipe Flow" en Trans. of the ASME. Moody cita el trabajo de Colebrook y White, entre otros, y propone en su investigación el famoso diagrama de Moody (1944). Este trabajo se ha tomado como base para muchos de los cálculos del factor de fricción en tuberías. Ha tenido propuestas de mejora, pero en esencia, ha perdurado con el tiempo la versión original del diagrama de Moody. Este diagrama se puede emplear como una solución gráfica de la ecuación de Colebrook. Sin embargo, hoy existen herramientas que permiten solucionar la ecuación de Colebrook tanto en su forma implícita como explícita, sin el uso del enfoque gráfico.

Un gran número de autores han destinado recursos y tiempo a resolver la ecuación implícita de Colebrook y han propuesto correlaciones explícitas que aproximan el resultado del factor de fricción con mucha precisión, además de simplificar su cálculo. Existen propuestas de tipo numéricas iterativas (Augusto et al., 2016), explícitas (Anaya et al., 2014) y algunas incluso han sido desarrolladas de manera simple y útil en hojas de cálculo (Brkic, 2017).

Como el resultado más relevante del trabajo de Colebrook, la ecuación para determinar el factor de fricción $(f)$ en tuberías para flujos en transición y turbulentos está relacionada con el valor de Re y $\varepsilon / D$ (rugosidad relativa de la tubería) como se presenta en la ecuación (1) (Colebrook, 1939):

$\frac{1}{\sqrt{f}}=-2 \log \left[\frac{\varepsilon / D}{3.7}+\frac{2.51}{\operatorname{Re} \sqrt{f}}\right]$

Donde $f$ es el factor de fricción; Re corresponde al Número de Reynolds; y $\varepsilon / \mathrm{D}$ a la rugosidad relativa de una tubería. A continuación se presenta la ecuación (2) que permite el cálculo de Re:

$$
\operatorname{Re}=\frac{\rho V D}{\mu}
$$

Donde $\mathrm{Q}, \mathrm{V}$ y $\mu$, corresponden a la densidad en $\left(\mathrm{kg}_{\text {masal }} /\right.$ $\left.\mathrm{m}^{3}\right)$, la velocidad media en $(\mathrm{m} / \mathrm{s})$ y la viscosidad diná- 
mica del fluido en (Pa s), respectivamente y $D$ al diámetro interno de la tubería en (m). Se puede observar claramente que la ecuación (1) es una expresión implícita que requiere una solución exacta o una solución numérica aproximada para estimar el factor de fricción. Por ejemplo, Anaya et al. (2014) evaluaron diferentes correlaciones explícitas que predicen el factor de fricción para un fluido en una tubería, mediante la comparación de valores numéricos de dichos factores respecto a la ecuación de Colebrook-White (Anaya et al., 2014). Winning y Coole (2013) en su estudio revisaron 28 ecuaciones explícitas para el cálculo del factor de fricción considerando tanto la precisión de la ecuación implícita de Colebrook-White y la eficiencia computacional de las ecuaciones explícitas.

En efecto, la ecuación (1) habitualmente es llamada ecuación de Colebrook-White, a pesar de que esta fue publicada solo por Colebrook en 1939 (Colebrook, 1939). Respecto a esta ecuación, Mikata y Walczak (2015) desarrollaron dos soluciones analíticas exactas de la ecuación de Colebrook-White, una por recursividad infinita y otra por el cálculo de una integral. En el caso de la recursividad infinita proponen una solución aproximada para la ecuación de Colebrook-White, llamada fórmula n-ésima. La clave para alcanzar esta solución, fue utilizar la forma cerrada de la expresión para una función asociada a la función $\mathrm{W}$ de Lambert, llamada función Y que se expresa por una recursión infinita que permite observar que el error absoluto disminuye geométricamente hacia cero dependiendo de la profundidad de la recursión. Además, dependiendo de la profundidad de recursión elegida para la fórmula n-ésima, la solución de esta puede estar por, sobre o debajo del valor exacto (Mikata y Walczak, 2015). Recientemente Mikata y Walczak, (2017) plantearon que existen tres tipos de soluciones para la ecuación de Colebrook-White. Las soluciones de primera generación corresponden a las aproximaciones basadas en ajustes de curvas con datos obtenidos de la ecuación Colebrook- White como por ejemplo las propuestas desarrolladas por Filonenko (1954), Papaevangelou, (2010), Buzzelli (2008), entre otros.

Las soluciones presentadas por Brkic (2011) para el factor de fricción, aplicando diferentes aproximaciones a la función W de Lambert (Brkic, 2011) corresponden a la solución formal de la ecuación de Colebrook-White. Estas aproximaciones de la función $\mathrm{W}$ de Lambert corresponden a una solución usando series de Taylor, la cual alcanza buenos resultados, la solución usando la función desplazada de Boyd, función que cuando la aproximación entregada se usa como primera solución a la función W, usando el método de Newton, a la cuarta iteración se reduce significativamente el error relativo respecto a la solución entregada (Boyd, 2017). Otra solución para la función $\mathrm{W}$ de Lambert es la propuesta por Barry y colaboradores la cual entrega valores de errores relativos cuando esta se aplica a la solución implícita de la ecuación de Colebrook-White, que se encuentra entre un 2.5 a $3 \%$ dependiendo de los valores de Re y $\varepsilon / D$ a estudiar (Barry et al., 2000). Finalmente se presenta otra aproximación a la función $\mathrm{W}$ de Lambert propuesta por Winitzki (2003) cuyos resultados son tan precisos como los presentados por Barry y colaboradores.

Por último, las soluciones de tercera generación son las aproximaciones basadas en soluciones analíticas exactas de la ecuación de Colebrook-White. La fórmula n-ésima pertenece a las soluciones de tercera generación y permite facilidad de uso y precisión en los resultados. Esta solución utiliza los mismos coeficientes que la ecuación de Colebrook-White, consiguiendo mejor precisión al aumentar la profundidad de recursión (n) (Mikata and Walczak, 2017).

Para resolver el valor del factor de fricción utilizando la fórmula n-ésima, es necesario hacer uso de herramientas computacionales que permitan calcular la profundidad de recursión para un valor de $n$ alto. Entre estas herramientas se encuentra VBA (Visual Basic para Aplicaciones) que es el lenguaje de programación de Excel con el que se pueden automatizar tareas escribiendo las llamadas macros, que se han utilizado en algunas aplicaciones de la mecánica de fluidos. Así, Zaragoza y Baeza (2003) utilizaron macros para la determinación del diámetro de sistemas de tuberías en flujo turbulento, considerando no solamente las pérdidas primarias, sino también las pérdidas menores vía la ecuación de Colebrook-White estableciendo un sistema de ecuaciones recurrentes. La solución de este sistema de ecuaciones fue implementada a partir de un algoritmo genérico en VBA con lo que se evitó el uso del diagrama de Moody (Zaragoza y Baeza, 2003). Le Roux y Brodalka (2004) también utilizaron VBA en el análisis de los perfiles de velocidad y su aplicación a estudios de trasporte de sedimentos en flujos constantes y uniformes en canal abierto. El programa requiere valores de entrada como la temperatura del agua, a partir de la cual se calculan la densidad y la viscosidad dinámica, profundidad y pendiente del canal, velocidades a diferentes alturas sobre el lecho, longitud y altura del mismo. Esta macros permite realizar procesos estandarizados en Excel, como es borrar la hoja de cálculo antes de analizar nuevos perfiles, actualizar las fórmulas, corregir los perfiles de velocidad, calcular el gradiente de velocidad y guardar los datos en una hoja separada para un análisis posterior (Le Roux y Brodalka, 2004). Por último, 
Duarte y Duarte (2015) establecieron el algoritmo computacional para el análisis nodal del flujo multifásico vertical en tuberías y presentaron el código implementado en Microsoft Excel VBA 2010, el cual además validaron mediante un software comercial.

Así, el objetivo del presente trabajo es la evaluación experimental de la solución analítica exacta de la ecuación de Colebrook-White determinada mediante el uso de macros de Excel y compararla con la solución numérica utilizada para predecir el factor de fricción en una tubería cilíndrica en flujo turbulento.

\section{Desarrollo}

\section{MÉTODOS Y PRUEBAS REALIZADAS}

Las mediciones se realizaron en un sistema construido dentro de un Laboratorio de Hidráulica. En las Figuras 1 y 2 se muestra el sistema para determinar el factor de fricción experimental $f_{\text {exp }}$ utilizando la ecuación (6) de Darcy-Weisbach. Este sistema consiste en una bomba centrífuga (REGGIO, STM 50), una tubería de PVC PN10 de $26 \mathrm{~mm}$ de diámetro interior y codos cortos de PVC de $90^{\circ}$, tees de PVC, dos conectores de acero para instalación de las conducciones del manómetro diferencial, válvulas de globo y un estanque graduado de descarga para medir el volumen. También se utilizó un manómetro diferencial (PSE-910) y un cronómetro (KENKO, KK-2802). Se midió el caudal usando el método volumétrico, para lo cual se realizaron 5 mediciones, y en cada una de ellas se verificó el largo o distancia entre los puntos de control de presión (L), junto con el diámetro interior (D) y la rugosidad $(\varepsilon)$ de la tubería. En el estanque acumulador de agua con control de volumen, se midió el tiempo (t) que demoraba en llenar 20 litros. De esta forma con la ecuación (3) se puede determinar la velocidad de escurrimiento al interior de la tubería.

$$
V=\frac{\left(\frac{V o l}{t}\right)}{\left(\frac{\pi \times D^{2}}{4}\right)}
$$

Donde $V$ es la velocidad de escurrimiento en (m/s), $V o l$ es el volumen igual a $0.02 \mathrm{~m}^{3}, t$ es el tiempo en (s) que demoró en llenar el volumen antes indicado, $D$ es el diámetro interior de la tubería igual a $0.026 \mathrm{~m}$ y $\pi$ es

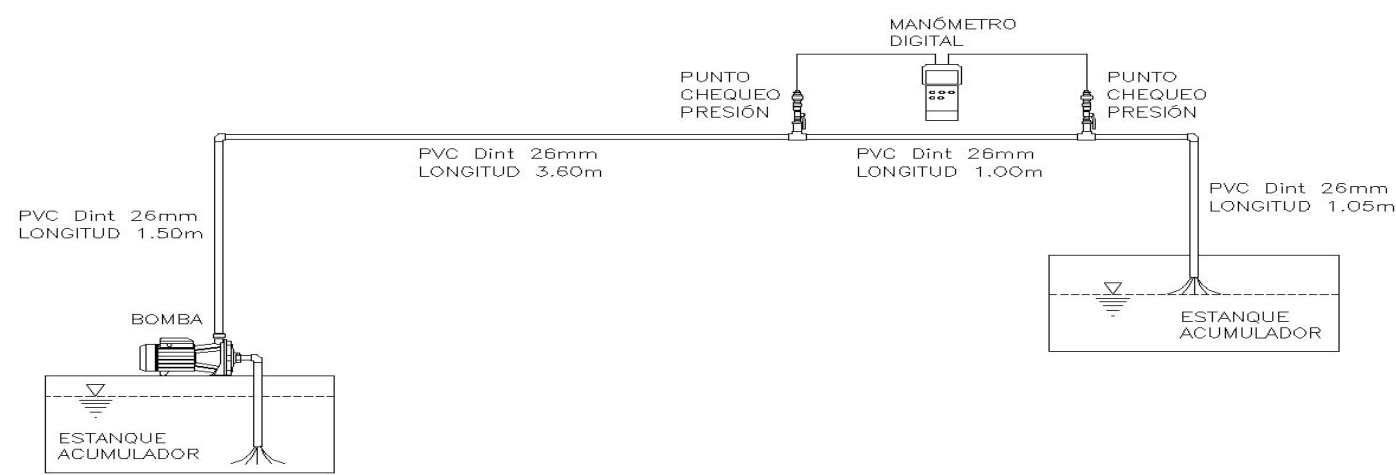

Figura 1. Esquema del sistema experimental para determinar el factor de fricción

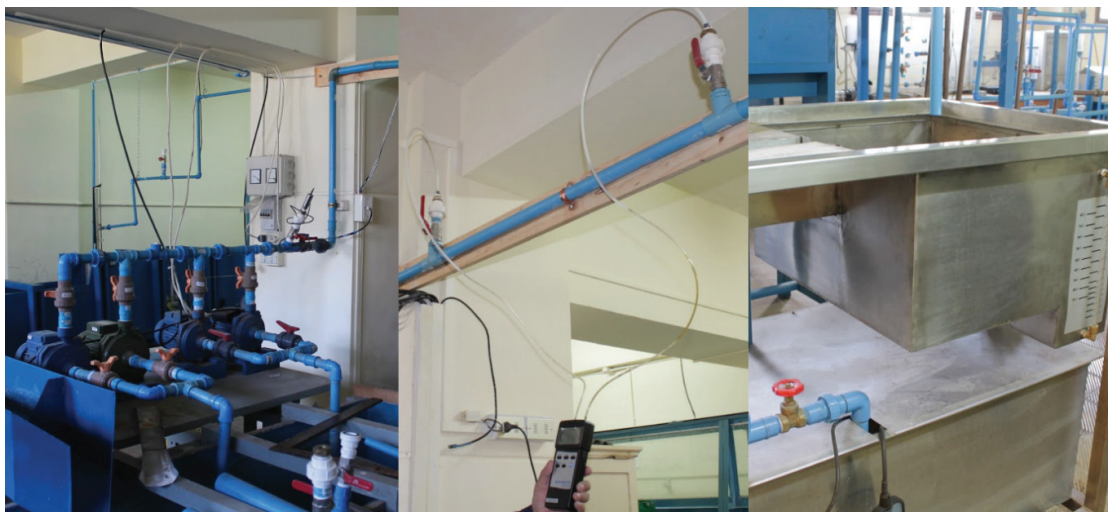

Figura 2. Fotografías del sistema experimental 
igual a 3.1416. Al mismo tiempo, se midió la diferencia de presión $(h)$ en el manómetro diferencial. Los valores obtenidos se reemplazan en la ecuación (4) para poder determinar $f_{\text {exp }}$

$f_{\exp }=\frac{h \times D \times 2 \times g}{L \times V^{2}}$

Donde $g$ es la aceleración de gravedad igual a $9.8\left(\mathrm{~m} / \mathrm{s}^{2}\right)$ y $L$ es la longitud de tubería recta entre los puntos de control de presión correspondiente a $1.0 \mathrm{~m}$.

\section{CÁlCULOS Y MODELOS MATEMÁTICOS}

SOlUCIÓN ANALítICA EXACTA DE LA ECUACIÓN DE COlebrook-White

Según lo planteado por Mikata y Walczak (2015), si se presenta la ecuación (1) de la forma en que se muestra en la ecuación (5).

$\frac{1}{\sqrt{f}}=c_{o}-c_{1} \operatorname{Ln}\left(c_{2}+\frac{c_{3}}{\sqrt{f}}\right)$

Con:

$c_{0}=0 \quad c_{1}=\frac{2}{\operatorname{Ln} 10} \quad c_{2}=\frac{\varepsilon / D}{3.7} \quad c_{3}=\frac{2.51}{R e}$

Si se considera la función $\mathrm{W}$ de Lambert definida en la ecuación (6) como:

$W e^{W}=x \Leftrightarrow W=W(x)$

Reordenando la ecuación (6) con las variables p, q y r que a continuación se presentan:

$p=\frac{1}{\sqrt{f}} \quad q=c_{2}+c_{3} p \quad r=\frac{q}{c_{1} c_{3}}$

Esta se trasforma en la ecuación (8).

$r e^{r}=\frac{1}{c_{1} c_{3}} e^{\left(1 / c_{1}\right)\left[c_{o}+\left(c_{2} / c_{3}\right)\right]}$

Y acomodando a la función W, se obtiene la ecuación (9).
$r(p)=W\left(\frac{1}{c_{1} c_{3}} e^{\left(1 / c_{1}\right)\left[c_{o}+\left(c_{2} / c_{3}\right)\right]}\right)$

Resolviendo la ecuación (9) para p definido en ecuación (7) se obtiene una solución exacta de la ecuación de Colebrook-White en términos de la función W de Lambert, que se presenta en la ecuación (10).

$\frac{1}{\sqrt{f}}=c_{1} W\left(\frac{1}{c_{1} c_{3}} e^{\left(1 / c_{1}\right)\left[c_{o}+\left(c_{2} / c_{3}\right)\right]}\right)-\frac{c_{2}}{c_{3}}$

Como el argumento de la función W de Lambert en la ecuación (10) es generalmente muy grande, es conveniente introducir otra función llamada función Y. Así la ecuación (10) se puede escribir de la siguiente forma:

$\frac{1}{\sqrt{f}}=c_{1} W\left(e^{\operatorname{Ln}\left(1 / c_{1} c_{3}\right)+\left(1 / c_{1}\right)\left[c_{0}+\left(c_{2} / c_{3}\right)\right]}\right)-\frac{c_{2}}{c_{3}}$

Donde la función Y está definida como:

$Y+\operatorname{Ln} Y=x \Leftrightarrow Y=Y(x)$

Esta función $\mathrm{Y}$ es exactamente la misma función que la función $\omega$ definida por Clamond (2009) y también por Lawrence y colaboradores (2012) donde la función se llama función Wright $\omega$ (Clamond, 2009; Lawrence et al., 2012). El nombre de la función se cambió porque la función W de Lambert, es a veces también llamada función omega (Clamond, 2009). Finalmente, de las ecuaciones (6) y (12) se obtiene lo siguiente:

$Y(x)=W\left(e^{x}\right)$

Usando la ecuación (13) en la ecuación (11) se obtiene:

$\frac{1}{\sqrt{f}}=c_{1} Y\left(x_{1}\right)-x_{o}$

donde:

$x_{o}=\frac{R e \cdot \varepsilon / D}{2.51 \cdot 3.7}$

$x_{1}=\operatorname{Ln}\left(\frac{\operatorname{Ln} 10 \cdot R e}{2 \cdot 2.51}\right)+\frac{\operatorname{Ln} 10 \cdot R e \cdot \varepsilon / D}{2 \cdot 2.51 \cdot 3.7}$

La ecuación 14 es una solución exacta de forma cerrada de la ecuación de Colebrook-White en términos de la 
función Y. Usando las ecuaciones (12) y (14) para un $\mathrm{x}_{1}$ mucho mayor que 1 , para rangos prácticos de $\operatorname{Re}$ y $\varepsilon / D$ como los del diagrama de Moody (Moody 1944), la solución de la ecuación de Colebrook-White obtenida es:

$$
\frac{1}{\sqrt{f}}=c_{1} Y\left(x_{1}\right)-x_{o} \quad\left(\mathrm{x}_{1}>1\right)
$$

Donde $\mathrm{x}_{0} \mathrm{y} \mathrm{x}_{1}$ fueron definidas anteriormente, además $\mathrm{Y}(\mathrm{x})$ está dado por la ecuación (13), por lo tanto tenemos:

$$
Y(x)=x-\operatorname{Ln}\{x-\operatorname{Ln}[x-\cdots-\operatorname{Ln}(x-\operatorname{Ln} x) \cdots]\}\left(\mathrm{x}_{1}>1\right)
$$

Finalmente, reemplazando la ecuación (18) en la ecuación (17) se obtienen las ecuaciones (19), (20), (21) y (22).

$$
\begin{aligned}
& \frac{1}{\sqrt{f_{1}}}=c_{1}\left[x_{2}-\operatorname{Ln}\left(x_{1}-\operatorname{Ln} x_{1}\right)\right] \\
& \frac{1}{\sqrt{f_{2}}}=c_{1}\left\{x_{2}-\operatorname{Ln}\left[\left(x_{1}-\operatorname{Ln}\left(x_{1}-\operatorname{Ln} x_{1}\right)\right]\right\}\right. \\
& \frac{1}{\sqrt{f_{3}}}=c_{1}\left(x_{2}-\operatorname{Ln}\left\{x_{1}-\operatorname{Ln}\left[x_{1}-\operatorname{Ln}\left(x_{1}-\operatorname{Ln} x_{1}\right)\right]\right\}\right) \\
& \frac{1}{\sqrt{f_{n}}}=c_{1}\left(x_{2}-\operatorname{Ln}\left\{x_{1}-\operatorname{Ln}\left[x_{1}-\operatorname{Ln}\left(x_{1}-\operatorname{Ln} x_{1}\right)\right] \cdots\right\}\right)
\end{aligned}
$$

Donde, $f_{n}$ es la enésima aproximación para el valor exacto de $f$, que corresponde al factor de fricción de Colebrook-White exacto (Solución analítica) (Mikata and Walczak, 2017)

\section{SOLUCIÓN NUMÉRICA DE LA ECUACIÓN DE Colebrook-White}

La ecuación (1) de Colebrook-White es ampliamente utilizada para determinar el factor de fricción para flujos turbulentos en tuberías rugosas. Se puede apreciar que la ecuación (1) se expresa de forma implícita para $f$ y requiere una solución iterativa para determinar su valor. El método adecuado para encontrar una raíz y que esta sea estable es el método de Newton. La solución aproximada del método se basa en el siguiente algoritmo:

$$
x_{i+1}=x_{i}-\frac{g\left(x_{i}\right)}{g^{\prime}\left(x_{i}\right)}
$$

En el método de Newton, la ecuación (23) se utiliza para calcular el factor de fricción a partir de un valor inicial dado y que permite estimar el siguiente valor de iteración, con el fin de encontrar la solución a la ecuación (1) del factor de fricción. La ecuación (1) de Colebrook-White puede reordenarse reemplazando el valor de $F=1 / \sqrt{ }$ y ser expresada mediante una nueva relación $\mathrm{g}(\mathrm{F})$ tal como se muestra en la ecuación (24).

$$
g(F)=F+2 \log \left[\frac{\varepsilon / D}{3.7}+\frac{2.51}{R e} \cdot F\right]
$$

Si se asume la ecuación (24) como una función que es siempre continua y diferenciable, se puede obtener la derivada de la función respecto a $F$, cuya deducción se presenta en el Anexo A y que se muestra en la ecuación (25).

$g^{\prime}(F)=1+\left[\frac{8.06659}{\left(\frac{\varepsilon}{D} \cdot R e+9.287 \cdot F\right)}\right]$.

La ecuación (25) tiene capacidad de rápida convergencia, particularmente si hay una estimación adecuada del valor inicial del factor de fricción (Augusto et al., 2016; Najafzadeh et al., 2018). En este trabajo la ecuación (1) se resolvió utilizando el método de Newton (Koçak, 2011).

\section{DisCuSIÓN Y ANÁlISIS DE RESULTADOS}

La Figura 3 muestra el gráfico de caja, que presenta la mediana (línea dentro de la caja), el promedio (cuadrado dentro de la caja), el primer cuartil (25\% de los datos), el tercer cuartil (el 75\% de los datos), el valor mínimo (5\% de los datos) y el valor máximo (95\% de los datos) del factor de fricción experimental medido en quintuplicado utilizando la ecuación (4), además se calculó su límite de confianza utilizando la ecuación (26).

La Figura 4 muestra la comparación de los resultados obtenidos para el cálculo de f, utilizando la solución analítica exacta y las diferentes propuestas mencionadas en este trabajo con su respectivo porcentaje de error respecto al valor experimental, presentando la correlación de Brkic el mayor porcentaje de error 
con $2.69 \%$, seguida del valor obtenido utilizando el gráfico de Moody con $0.58 \%$, luego Filonenko con $0.42 \%$ y las soluciones de la W de Lambert de Winitzki y Boyd con $0.33 \%$ y $0.10 \%$, respectivamente. Finalmente las correlaciones de Papaevangelou con $0.06 \%$, Buzzelli y Colebrook-White con $0.01 \%$, la solución de la $W$ de Lambert de Barry con $0.001 \%$ y la solución analítica exacta con un $0.0000 \%$ son las que presentaron los menores porcentajes de error.

A continuación se muestra la ecuación (26) para el cálculo del límite de confianza:

L.C. $=f_{\text {exp }, \text { prom }}+\frac{t_{95 \%} \cdot s}{\sqrt{n}}$

donde

L.C. = límite de confianza

$f_{\text {exp,prom }}=$ factor de fricción promedio

$t_{95 \%} \quad=$ valor $t$ de Student para $n-1$ grados de libertad a $95 \%$ de confianza

s = desviación estándar

$n \quad=$ número de réplicas

En este caso el L.C. es igual a $0.02242677 \pm 0.002190$. Cada uno de los valores del factor de fricción calculados mediante la fórmula n-ésima y la solución numérica de Newton fueron evaluados y comparados respecto

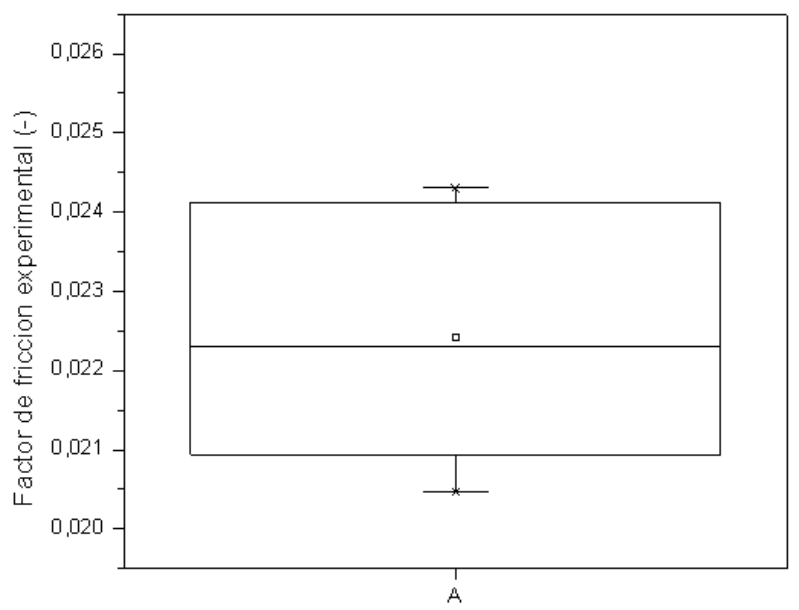

Figura 3. Gráfico de caja del factor de fricción experimental a su error relativo porcentual con el factor de fricción experimental promedio, los que se muestran en la Tabla 1. El error relativo porcentual se calculó utilizando la ecuación (27):

$\% E=\left|\frac{f_{\text {exp }, \text { prom }}-f_{\text {pred }}}{f_{\text {exp }, \text { prom }}}\right| \times 100$

Donde $f_{\text {pred }}$ es el factor de fricción obtenido para la fórmula n-ésima y para la solución numérica de Newton indicadas en la Tabla 1, considerando una rugosidad $(\varepsilon)$ de $0.0015 \mathrm{~mm}$ para PVC (Neto y Porto, 2004) y $\varepsilon / D$ (rugosidad relativa de la tubería) igual a 0.0000576923 . En el caso de la correlación de Colebrook-White, el factor de fricción se obtuvo utilizando un programa en $\mathrm{C}++$ para el método numérico de Newton y una macro de Excel para evaluar la fórmula n-ésima, la cual se presenta en la Figura 5.

La Tabla 1 muestra los resultados obtenidos para el factor de fricción con cada una de las aproximaciones de la fórmula n-ésima obtenidas con la macro de Excel y el error relativo porcentual respectivo. El estudio se basa en la evaluación experimental del valor del factor de fricción, encontrándose que el aumento en la profundidad de recursión aproxima de mejor manera el valor obtenido por la ecuación de Colebrook-White para un rango de valores de Número de Reynolds de baja turbulencia, comprendido entre $37.079 \leq \operatorname{Re} \leq 38.703$.

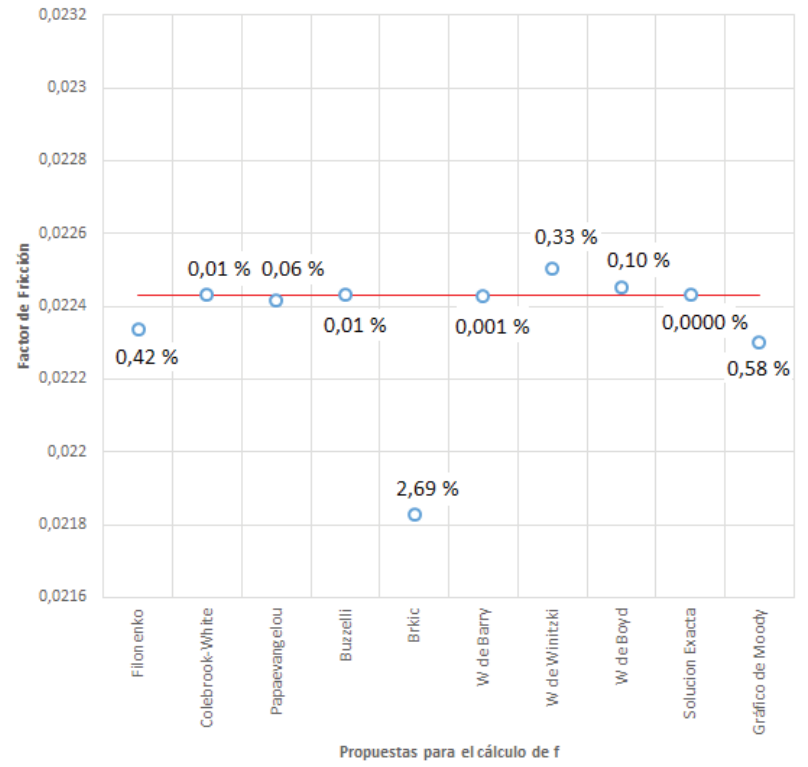

Figura 4. Gráfico que presenta los resultados de diferentes propuestas de cálculo para el factor de fricción. La línea roja representa el valor experimental 
Sub Mikata20150

Dim n, fila, Error1, Error2 As Integer

Dim Re, ED, C1, t, X2, X1, X3, F, Factor, Error, E1, E2 As Double

'Las variables X1, X2 y X3 no guardan relación con las variables x 0 y x 1

'del texto principal y solo se utilizan para la resolución numérica interna de la subrutina

'Lectura de variables Re, E/D y n desde el Formulario

'Cálculo de Constantes y variables para la determinación de f

$$
\begin{aligned}
& \mathrm{Re}=\text { Range("C5").Value } \\
& \mathrm{ED}=\text { Range("C7").Value } \\
& \mathrm{n}=\text { Range("C9").Value } \\
& \mathrm{C} 1=2 / \log (10) \\
& \mathrm{t}=(1 / \mathrm{C} 1) * \operatorname{Re} / 2.51 \\
& \mathrm{X} 2=\log (\mathrm{t}) \\
& \mathrm{X} 1=\mathrm{X} 2+\mathrm{t} * \mathrm{ED} / 3.7 \\
& \mathrm{X} 3=\mathrm{X} 1
\end{aligned}
$$

'Ciclo para el cálculo de Factor de fricción

'Fórmula n-ésima

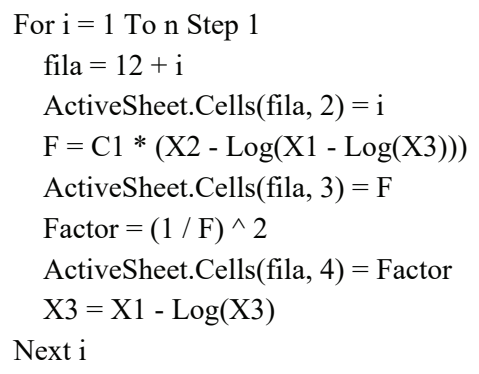

'Ciclo para el cálculo del error relativo de f

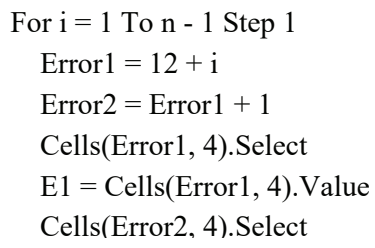

La Tabla 1 muestra el valor del factor de fricción con 15 decimales lo que solo tiene por objetivo resaltar la diferencia numérica entre los distintos valores calculados en función de la profundidad de la recursión $n$.

La Figura 6 muestra la gráfica de los valores del error relativo porcentual asociado a la profundidad de la recursión, donde se puede apreciar la oscilación del valor del error relativo porcentual respecto a un valor constante del error. Este valor constante representa el error relativo porcentual del valor experimental versus la solución exacta de la fórmula n-ésima, lo cual es concordante con lo plateado por Mikata y Walczak (2017), en lo que respecta a la forma de oscilación del error.
Figura 5. Código de Macro en Excel para evaluar la fórmula n-ésima del factor de fricción

El método de Newton es uno de los métodos clásicos utilizados para la determinación numérica de ecuaciones no lineales y de fácil aplicación y rapidez en su implementación. Como se indicó para dicha resolución se utilizó un programa en $\mathrm{C}++$, cuya salida de datos se entrega en la Tabla 2.

El resultado de $X i$ corresponde a $F=1 / \sqrt{ } f$, con el que se calcula el valor para el factor de fricción obtenido mediante la solución numérica de Newton de la ecuación de Colebrook-White y que presenta un valor de 0.0224321 , por lo que este valor genera un error relativo porcentual de $0.02378591 \%$. 
Tabla 1. Factor de fricción y error relativo porcentual de la solución analítica exacta de la ecuación de Colebrook-White

\begin{tabular}{|c|c|c|}
\hline Profundidad de la Recursión (n) & Factor de fricción & Error relativo porcentual \% \\
\hline 1 & 0,0222606710084215 & 0,7416462 \\
\hline 2 & 0,0224537397748318 & 0,1192303 \\
\hline 3 & 0,0224293785322303 & 0,0106057 \\
\hline 4 & 0,0224324384636314 & 0,0242496 \\
\hline 5 & 0,0224320538962749 & 0,0225349 \\
\hline 6 & 0,0224321022246198 & 0,0227504 \\
\hline 7 & 0,0224320961511713 & 0,0227233 \\
\hline 8 & 0,0224320969144239 & 0,0227267 \\
\hline 9 & 0,0224320968185056 & 0,0227263 \\
\hline 10 & 0,0224320968305597 & 0,0227263 \\
\hline 11 & 0,0224320968290449 & 0,0227263 \\
\hline 12 & 0,0224320968292352 & 0,0227263 \\
\hline 13 & 0,0224320968292113 & 0,0227263 \\
\hline 14 & 0,0224320968292143 & 0,0227263 \\
\hline 15 & 0,0224320968292139 & 0,0227263 \\
\hline 16 & 0,0224320968292140 & 0,0227263 \\
\hline 17 & 0,0224320968292140 & 0,0227263 \\
\hline 18 & 0,0224320968292140 & 0,0227263 \\
\hline 19 & 0,0224320968292140 & 0,0227263 \\
\hline 20 & 0,0224320968292140 & 0,0227263 \\
\hline 21 & 0,0224320968292140 & 0,0227263 \\
\hline
\end{tabular}

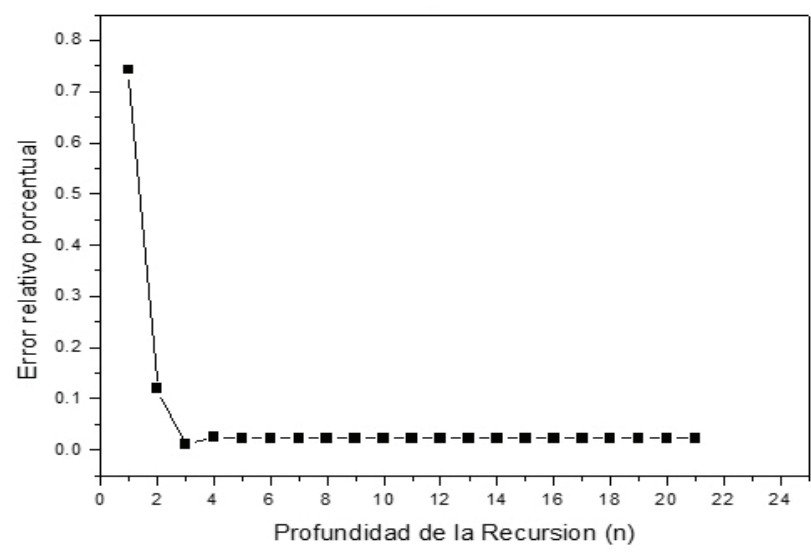

Figura 6. Valor de la recursión escogida (n) versus el porcentaje de error 
Tabla 2. Resultados iteración método de Newton para el factor de fricción

\begin{tabular}{cccc}
\hline$i$ & $X i$ & $G(X i)$ & Error de Iteración \\
\hline 0 & 4.83854 & -210.6770000 & 5.065880000 \\
1 & 6.62467 & -0.058647900 & 2.048120000 \\
2 & 6.67652 & -0.000257486 & 0.058390400 \\
3 & 6.67675 & $-1.00790 \mathrm{E}-06$ & 0.000256478 \\
4 & 6.67675 & $-3.94323 \mathrm{E}-09$ & $1.00 \mathrm{E}-06$ \\
5 & 6.67675 & $-1.54268 \mathrm{E}-11$ & $3.93 \mathrm{E}-09$ \\
\hline
\end{tabular}

\section{Conclusiones}

En el presente trabajo se construyó un sistema hidráulico para la determinación experimental del factor de fricción en una tubería de PVC en flujo turbulento, el cual permitió evaluar de forma experimental el factor de fricción comparándolo con el obtenido por medio de la fórmula n-ésima como aproximación de la solución exacta de la ecuación de Colebrook-White, las correlaciones de Papaevangelou, Buzzelli, Brkic, las soluciones basadas en la W de Lambert de Barry, Winitzki y Boyd y el valor obtenido utilizando el grafico de Moody presentaron un porcentaje de error mayor que el alcanzado empleando la solución analítica exacta. La fórmula n-ésima predijo el factor de fricción con un error relativo que tiende a converger dependiendo de la profundidad de la recursión utilizando una macros de Excel en lenguaje VBA.

El aporte principal del presente trabajo fue la evaluación de la fórmula n-ésima del factor de fricción y su comparación con los resultados experimentales obtenidos en el laboratorio y a los obtenidos por otros autores utilizando diferentes metodologías de cálculo, donde se puede destacar que la solución exacta entrega valores muy similares a las propuestas de Filonenko (1954), Papaevangelou et al. (2010), Buzzelli (2008) y función W de Lambert creada por Winitzki (2003) y Boyd (2017), lo que permitió probar la validez de la predicción de la fórmula con un caso real, con un error relativo porcentual menor al predicho por la solución numérica de Colebrook-White.

\section{Anexo A}

\section{Complemento derivada ecuación Colebrook-White}

Para la determinación del factor de fricción en flujos turbulentos para tuberías rugosas se utiliza la ecuación (A.1) de Colebrook-White. Esta relación tiene una forma implícita para el valor de $f$, tal como sigue:

$$
\frac{1}{\sqrt{f}}=-2 \log \left[\frac{2.51}{R e \sqrt{f}}+\frac{\varepsilon / D}{3.7}\right]
$$

donde

$\mathrm{f}=$ factor de fricción de la tubería

$\mathrm{Re}=$ número de Reynolds

$\varepsilon / \mathrm{D}=$ rugosidad relativa de la tubería

Si se reescribe la ecuación (A.1) reemplazando el valor $\mathrm{F}=1 / \sqrt{ } \mathrm{f}$ y luego despejando la expresión, se tiene:

$g(F)=F+2 \log \left[\frac{2.51}{\operatorname{Re}} F+\frac{\varepsilon / D}{3.7}\right]$

Derivando de manera implícita la ecuación (A.2) respecto a la variable $F$, se obtiene lo siguiente:

$$
\frac{d(g(F)}{d F}=g^{\prime}(F)=1+2 \cdot \frac{\mathrm{d}}{\mathrm{dF}}\left(\log \left[\frac{\varepsilon / D}{3.7}+\frac{2.51}{R e} \cdot F\right]\right)
$$

Utilizando la derivada implícita para F se tiene que:

$$
\frac{d(\log u)}{d F}=\frac{u^{\prime}}{u} \cdot \frac{1}{\ln 10}
$$

Por lo tanto, la derivada de la función $g(F)$ respecto a $F$ es:

$$
g^{\prime}(F)=1+2 \cdot\left[\frac{\frac{2.51}{R e}}{\left(\frac{\varepsilon / D}{3.7}+\frac{2.51}{R e} \cdot F\right)} \frac{1}{\ln 10}\right]
$$

Reescribiendo la expresión anterior se tiene: 


$$
g^{\prime}(F)=1+\left[\frac{8.06659}{\varepsilon / D \cdot R e+9.287 \cdot F}\right]
$$

La ecuación (A.6) es la derivada de la función $g(F)$ que se ha empleado en el programa desarrollado en lenguaje C++ y que permite iterar y encontrar la solución numérica del factor de fricción para la ecuación (A.1) de Colebrook-White (Augusto et al., 2016; Najafzadeh et al., 2018)

\section{RefERenCias}

Anaya, A.I., Cauich, G.I., Funabazama, O., Gracia, V.A. (2014). Evaluación de ecuaciones de factor de fricción explícito para tuberías. Educación Química, 25(2), 128-134. https://doi. org/10.1016/S0187-893X(14)70535-X

Ángeles, M.S., Chéret, I., et al. (2000). Manejo integrado de recursos hídricos.

Augusto, G.L., Culaba, A.B., Tanhueco, R.M.T. (2016). Pipe sizing of District cooling distribution network using implicit Colebrook-White equation. Journal of Advanced Computational Intelligence and Intelligent Informatics, 20(1), 76-83. https://doi. org/10.20965/jaciii.2016.p0076

Barry, D. ., Parlange, J.-Y., Li, L., Prommer, H., Cunningham, C. ., Stagnitti, F. (2000). Analytical approximations for real values of the Lambert W-function. Mathematics and Computers in Simulation, 53(1), 95-103. https://doi.org/10.1016/S03784754(00)00172-5

Boyd, J.P. (2017). New approximations to the principal real-valued branch of the Lambert W-function. Advances in Computational Mathematics, 43(6), 1403-1436. https://doi.org/10.1007/ s10444-017-9530-3

Brkic, D. (2011). W solutions of the CW equation for flow friction. Applied Mathematics Letters, 24(8), 1379-1383. https://doi. org/10.1016/j.aml.2011.03.014

Brkic, D. (2017). Solution of the implicit colebrook equation for flow friction using excel. Spreadsheets in Education (EJSiE), 10(2), 1.12.

Buzzelli, D. (2008). Calculating friction in one step. Machine Design, 80(12), 54-55.

Clamond, D. (2009). Efficient resolution of the colebrook equation. Industrial and Engineering Chemistry Research, 48(7), 3665-3671. https://doi.org/10.1021/ie801626g

Colebrook, C.F. (1939). Turbulent flow in pipes, with particular reference to the transition region between the smooth and rough pipe Laws. Journal of the Institution of Civil Engineers, 12(8), 393-422. https://doi.org/10.1680/ijoti.1939.14509

Colebrook, C.F., \& White, C.M. (1937). Experiments with fluid friction in roughened pipes. proceedings of the royal society. Mathematical, Physical and Engineering Sciences, 161(906), 367381. https://doi.org/10.1098/rspa.1937.0150
Duarte, L., \& Duarte, M. (2015). Algoritmo computacional para predecir el gradiente de presión en pozos verticales mediante la correlación de flujo multifásico de hagedorn y brown. Telémtique, 14(2), 153-174.

Filonenko, G. (1954). Hydraulic resistance in pipes. Teploenergetika, 1, 40-44.

Koçak, M.Ç. (2011). Ostrowski's fourth-order iterative method speedily solves cubic equations of state. Journal of Computational and Applied Mathematics, 235(16), 4736-4741. https://doi. org/10.1016/j.cam.2010.09.012

Lawrence, P.W., Corless, R.M., Jeffrey, D.J. (2012). Algorithm 917. ACM Transactions on Mathematical Software, 38(3), 1-17. https:// doi.org/10.1145/2168773.2168779

Le Roux, J.P. \& Brodalka, M. (2004). An ExcelTM-VBA programme for the analysis of current velocity profiles. Computers and Geosciences, 30(8), 867-879. https://doi.org/10.1016/j.cageo.2004.06.006

Mikata, Y. \& Walczak, W.S. (2015). Exact analytical solutions of the colebrook-white equation. Journal of Hydraulic Engineering, 142(2), 04015050. https://doi.org/10.1061/(ASCE)HY.19437900.0001074

Mikata, Y. \& Walczak, W.S. (2017). Closure to "Exact Analytical Solutions of the Colebrook-White Equation". Journal of Hydraulic Engineering, 143(9), 07017008. https://doi.org/10.1061/ (ASCE)HY.1943-7900.0001074

Moody, L.F. (1944). Friction factors for pipe flow. Trans. Asme, 66, 671-684.

Najafzadeh, M., Shiri, J., Sadeghi, G., Ghaemi, A. (2018). Prediction of the friction factor in pipes using model tree. ISH Journal of Hydraulic Engineering, 24(1), 9-15. https://doi.org/10.1080 /09715010.2017.1333926

Neto, L. \& Porto-R. D.M. (2004). Performance of Low-Cost Ejectors, (April), 122-128.

Özger, M. \& Yildirim, G. (2009). Determining turbulent flow friction coefficient using adaptive neuro-fuzzy computing technique. Advances in Engineering Software, 40(4), 281-287. https:// doi.org/10.1016/j.advengsoft.2008.04.006

Papaevangelou, G., Evangelides, C., Tzimopoulos, C. (2010). A new explicit relation for friction coefficient $f$ in the DarcyWeisbach equation. Protection and Restoration of the Environment, Corfu, 166-173.

White Frank, M. (2008). Mecánica de fluidos. México: Mc Graw Hill. Winitzki, S. (2003). Uniform Approximations for transcendental functions, 780-789. https://doi.org/10.1007/3-540-44839-X_82

Winning, H.K., \& Coole, T. (2013). Explicit friction factor accuracy and computational efficiency for turbulent flow in pipes. Flow, Turbulence and Combustion, 90(1), 1-27. https://doi. org/10.1007/s10494-012-9419-7

Zaragoza, N., \& Baeza, J.R. (2003). Determinación del diámetro de sistemas de tuberías mediante la utilización del visual basic para aplicaciones y el método de aproximación de punto fijo. Ingeniería, 2, 55-64. 